\title{
Magnetoconductance properties of rectangular arrays of spintronic quantum rings
}

\author{
Orsolya Kálmán, ${ }^{1,2}$ Péter Földi, ${ }^{2}$ Mihály G. Benedict, ${ }^{2, \text { 冈 }}$ and F. M. Peeters ${ }^{3}$ \\ ${ }^{1}$ Department of Nonlinear and Quantum Optics, Research Institute for Solid State Physics and Optics, \\ Hungarian Academy of Sciences, Konkoly-Thege Miklós út 29-33, H-1121 Budapest, Hungary \\ ${ }^{2}$ Department of Theoretical Physics, University of Szeged, Tisza Lajos körút 84, H-6720 Szeged, Hungary \\ ${ }^{3}$ Departement Fysica, Universiteit Antwerpen, Groenenborgerlaan 171, B-2020 Antwerpen, Belgium
}

\begin{abstract}
Electron transport through multi-terminal rectangular arrays of quantum rings is studied in the presence of Rashba-type spin-orbit interaction (SOI) and of a perpendicular magnetic field. Using the analytic expressions for the transmission and reflection coefficients for single rings we obtain the conductance through such arrays as a function of the SOI strength, the magnetic flux, and of the wave vector $k$ of the incident electron. Due to destructive or constructive spin interferences caused by the SOI, the array can be totally opaque for certain ranges of $k$, while there are parameter values where it is completely transparent. Spin resolved transmission probabilities show nontrivial spin transformations at the outputs of the arrays. When point-like random scattering centers are placed between the rings, the Aharonov-Bohm peaks split, and an oscillatory behavior of the conductance emerges as a function of the SOI strength.
\end{abstract}

PACS numbers: 03.65.-w, 85.35.Ds, 71.70.Ej, 73.23.Ad

\section{INTRODUCTION}

Magnetoconductance oscillations of quantum rings made of semiconducting materials 1 exhibiting Rashbatype spin-orbit interaction ${ }^{2.3 .4}$ (SOI) have been intensely studied in the past few years. These effects are manifestations of flux- and spin-dependent quantum interference phenomena. In view of the possible spintronic applications and the conceptual importance of these interference effects in multiply-connected domains, closed single quantum rings (without attached leads), $,+6,7.8$ as well as two- or three-terminal ones were investigated $9,10,11,12,13,14,15,16,17,18,19,20,21,22$ extensively. Additionally, the conductance properties of a linear chain of rings have also been determined $\underline{23}$

In this paper we present a method that enables one to calculate the conductance and the spin transport properties of two-dimensional rectangular arrays consisting of quantum rings with Rashba-type $\mathrm{SOI}^{24}$ and with a perpendicular magnetic field. Such arrays, fabricated from e.g. an InAlAs/InGaAs based $2 \mathrm{DEG}, \underset{25}{,}$ have been studied in a recent experiment $\underline{26}$ and in a subsequent theoretical work ${ }^{27}$ to demonstrate the time-reversal AharonovCasher effect $\underline{28}$ Here we present a more general survey of the magnetoconductance properties of such devices, including the perturbative treatment of the magnetic field which still allows us to analytically solve the scattering problem in case of two-, three- and four-terminal rings, which are then used as building blocks of larger arrays. Our method is based on analytic results, and can be used for an arbitrary configuration. For the sake of definiteness, we consider $3 \times 3,4 \times 4$ and $5 \times 5$ rectangular arrays,$\frac{26,27}{,}$ which are closed in the vertical, and open in the horizontal direction. Additionally, we study the magnetoconductance properties and spin resolved transmission probabilities of the same array geometry with only one input channel. We also investigate to what extent the conductance properties are modified by the presence of point-like random scattering centers between the rings. In our calculations we assume that the rings are narrow enough to be considered one-dimensional and the transport of the electrons through the arrays is ballistic. We determine the magnetoconductance in the framework of the Landauer-Büttiker formalism. 29

Rectangular arrays 26,27 -depending on the number of input leads- consist of two-, three- and four-terminal rings (see Fig. 10, where the two- and three-terminal ones are situated on the boundary of the arrays as shown in Fig. 2 with or without the input leads displayed by dashed lines. The transmission and reflection properties of two- and three-terminal rings have been determined in previous works $10,11,12,14,30,31,32,33,34,35$ but the effect of the magnetic field on the spin degree of freedom has not been taken into account for an arbitrary geometry. Additionally, the most general boundary condition that is required by this two-dimensional problem has not been investigated so far. Therefore in Sec. [II we first consider a perpendicular magnetic field as a weak perturbation, then, in order to account for all possible reflections and transmissions when building up the array from single rings, we generalize our previous results to the case when electrons can enter/exit on any of the terminals of a three-terminal ring (results for two- and four-terminal rings are presented in the Appendix). Next, in Sec. III A the individual rings are used as building blocks of the arrays by fitting the wave functions and their derivatives in the points where neighboring rings touch each other. Magnetoconductance properties are presented here as a function of the wave number $k$ of the incoming electron, the magnetic flux and the SOI strength. Spin resolved transmission probabilities on the output side of the arrays are also derived. In Sec. IIIB we investigate the effect of random Dirac-delta scattering potentials in between the rings. 


\section{BUILDING BLOCKS OF TWO-DIMENSIONAL ARRAYS: SINGLE QUANTUM RINGS}

In this section we consider a single, narrow quantum ring 31 of radius $a$ located in the $x y$ plane in the presence of Rashba $\mathrm{SOI}^{24}$ and a perpendicular magnetic field $B$. If $B$ is relatively weak, then the interaction between the electron spin and the field, i.e. the Zeeman term can be treated as a perturbation and the relevant dimensionless Hamiltonian reads 11,36

$$
H=\left[\left(-i \frac{\partial}{\partial \varphi}-\frac{\Phi}{\Phi_{0}}+\frac{\omega_{\mathrm{SO}}}{2 \Omega} \sigma_{r}\right)^{2}-\frac{\omega_{\mathrm{SO}}^{2}}{4 \Omega^{2}}\right]+H_{\mathrm{p}}
$$

where $\varphi$ is the azimuthal angle of a point on the ring, $\Phi$ denotes the magnetic flux encircled by the ring, $\Phi_{0}=h / e$ is the unit flux, and $\omega_{\mathrm{SO}}=\alpha / \hbar a$ is the frequency associated with the spin-orbit interaction. $\hbar \Omega=\hbar^{2} / 2 m^{*} a^{2}$ characterizes the kinetic energy with $m^{*}$ being the effective mass of the electron, and the radial spin operator is given by $\sigma_{r}=\sigma_{x} \cos \varphi+\sigma_{y} \sin \varphi$. The perturbative term $H_{\mathrm{p}}$ is given by 11

$$
H_{\mathrm{p}}=\frac{\omega_{\mathrm{L}}}{\Omega} \sigma_{z}
$$

where $\omega_{\mathrm{L}}=g^{*} e B / 4 m$ with $g^{*}$ and $m$ being the effective gyromagnetic ratio and the free electron mass, respectively.

The energy eigenvalues of the unperturbed Hamiltonian are

$$
E_{0}^{(\mu)}(\kappa)=\left(\kappa-\frac{\Phi}{\Phi_{0}}\right)^{2}+(-1)^{\mu}\left(\kappa-\frac{\Phi}{\Phi_{0}}\right) w+\frac{1}{4},(\mu=1,2)
$$

and the corresponding eigenvectors in the $\left|\uparrow_{z}\right\rangle,\left|\downarrow_{z}\right\rangle$ eigenbasis of $\sigma_{z}$ read

$$
\psi^{(\mu)}(\kappa, \varphi)=e^{i \kappa \varphi}\left(\begin{array}{c}
e^{-i \varphi / 2} u^{(\mu)} \\
e^{i \varphi / 2} v^{(\mu)}
\end{array}\right)
$$

where $u^{(1)}=-v^{(2)}=\cos (\theta / 2) ; u^{(2)}=v^{(1)}=\sin (\theta / 2)$ and

$$
\tan (\theta / 2)=\frac{\Omega}{\omega_{\mathrm{SO}}}(1-w)
$$

with $w=\sqrt{1+\omega_{\mathrm{SO}}^{2} / \Omega^{2}}$.

The matrix elements of $H_{\mathrm{p}}$ in the basis of these eigenstates are obtained as

$$
\begin{gathered}
\left\langle\psi^{(\mu)}\left|H_{\mathrm{p}}\right| \psi^{(\mu)}\right\rangle=(-1)^{\mu+1} \frac{\omega_{\mathrm{L}}}{\Omega} \cos \theta=(-1)^{\mu+1} \frac{\omega_{\mathrm{L}}}{\Omega} \frac{1}{w}, \\
\left\langle\psi^{(1)}\left|H_{\mathrm{p}}\right| \psi^{(2)}\right\rangle=\frac{\omega_{\mathrm{L}}}{\Omega} \sin \theta .
\end{gathered}
$$

In the first-order approximation one neglects the offdiagonal elements; this is reasonable if they are small, i.e., if $\omega_{\mathrm{L}} / \Omega \ll k^{2} a^{2}$, where $k$ denotes the wave number of the incident electron, which is described as a plane wave. Within this approximation, the eigenspinors are not perturbed and their direction is still specified by the angle $\theta$ given by Eq. (3). The energy eigenvalues including the first-order corrections are given by

$$
E^{(\mu)}(\kappa)=E_{0}^{(\mu)}(\kappa)+(-1)^{\mu+1} \frac{\omega_{\mathrm{L}}}{\Omega} \frac{1}{w} .
$$

Imposing the condition of energy conservation $k^{2} a^{2}=$ $E^{(\mu)}(\kappa)$ determines the possible values of $\kappa$ :

$$
\kappa_{j}^{(\mu)}=(-1)^{\mu+1}\left[\frac{w}{2}+(-1)^{j} q^{(\mu)}\right]+\frac{\Phi}{\Phi_{0}},
$$

where $\mu, j=1,2$ and

$$
q^{(\mu)}=\sqrt{q^{2}+(-1)^{\mu} \frac{\omega_{L}}{\Omega} \frac{1}{w}}
$$

with $q=\sqrt{\left(\omega_{\mathrm{SO}} / 2 \Omega\right)^{2}+E / \hbar \Omega}$, and $E=\hbar^{2} k^{2} / 2 m^{*}$ denoting the energy of the incoming electron. The corresponding four eigenspinors read

$$
\begin{aligned}
& \psi_{j}^{(1)}\left(\kappa_{j}^{(1)}, \varphi\right)=e^{i \kappa_{j}^{(1)} \varphi}\left(\begin{array}{c}
e^{-i \varphi / 2} \cos (\theta / 2) \\
e^{i \varphi / 2} \sin (\theta / 2)
\end{array}\right), \\
& \psi_{j}^{(2)}\left(\kappa_{j}^{(2)}, \varphi\right)=e^{i \kappa_{j}^{(2)} \varphi}\left(\begin{array}{c}
e^{-i \varphi / 2} \sin (\theta / 2) \\
-e^{i \varphi / 2} \cos (\theta / 2)
\end{array}\right) .
\end{aligned}
$$

The wave functions belonging to the same energy in the different sections of the ring are linear combinations of these eigenspinors.

The building blocks of the rectangular arrays we investigate are two-, three- and four-terminal quantum rings (see Fig. 1), where, in general, the boundary conditions allow both incoming and outgoing spinor valued wave functions at each terminal: $\Psi_{i}=f_{i} e^{i k x_{i}}+r_{i} e^{-i k x_{i}}$ $(i=I, I I, I I I, I V)$, where $x_{i}$ denotes the local coordinate in terminal $i$. Note that the amplitudes $f_{I}, r_{I}, f_{I I}$, etc. refer to two-component spinors, e.g. $f_{I}=\left(\begin{array}{l}\left(f_{I}\right)_{\uparrow} \\ \left(f_{I}\right)_{\downarrow}\end{array}\right)$. For the sake of definiteness, we focus on a general threeterminal ring, shown in Fig. 1(b). The scattering problem in the case of a ring with four terminals (Fig. 1(c) can also be solved analytically, as presented in the Appendix, where we also give the results for a general two-terminal ring (Fig. 1(a)). The outgoing spinors $\left(r_{i}, i=I, I I, I I I\right)$ are connected to the incoming ones $\left(f_{i}\right)$ by $2 \times 2$ matrices, which can be determined by requiring the continuity of the wave functions, and vanishing net spin current densities (Griffith conditions) ${ }^{11,13,32,37}$ at the junctions. For the same boundary conditions as in Ref. 35, i.e. for $f_{I I}, f_{I I I}=0$ in Fig. $1(\mathrm{~b})$, the reflection matrix which connects $r_{I}$ to the incoming spinor $f_{I}$ is given by

$$
\begin{aligned}
R_{\uparrow \uparrow}^{f_{I}} & =\varrho^{(1)} \cos ^{2}(\theta / 2)+\varrho^{(2)} \sin ^{2}(\theta / 2)-1, \\
R_{\uparrow \downarrow}^{f_{I}} & =\left(\varrho^{(1)}-\varrho^{(2)}\right) \sin (\theta / 2) \cos (\theta / 2), \\
R_{\downarrow \uparrow}^{f_{I}} & =R_{\uparrow \downarrow}^{f_{I}}, \\
R_{\downarrow \downarrow}^{f_{I}} & =\varrho^{(1)} \sin ^{2}(\theta / 2)+\varrho^{(2)} \cos ^{2}(\theta / 2)-1,
\end{aligned}
$$


(a)
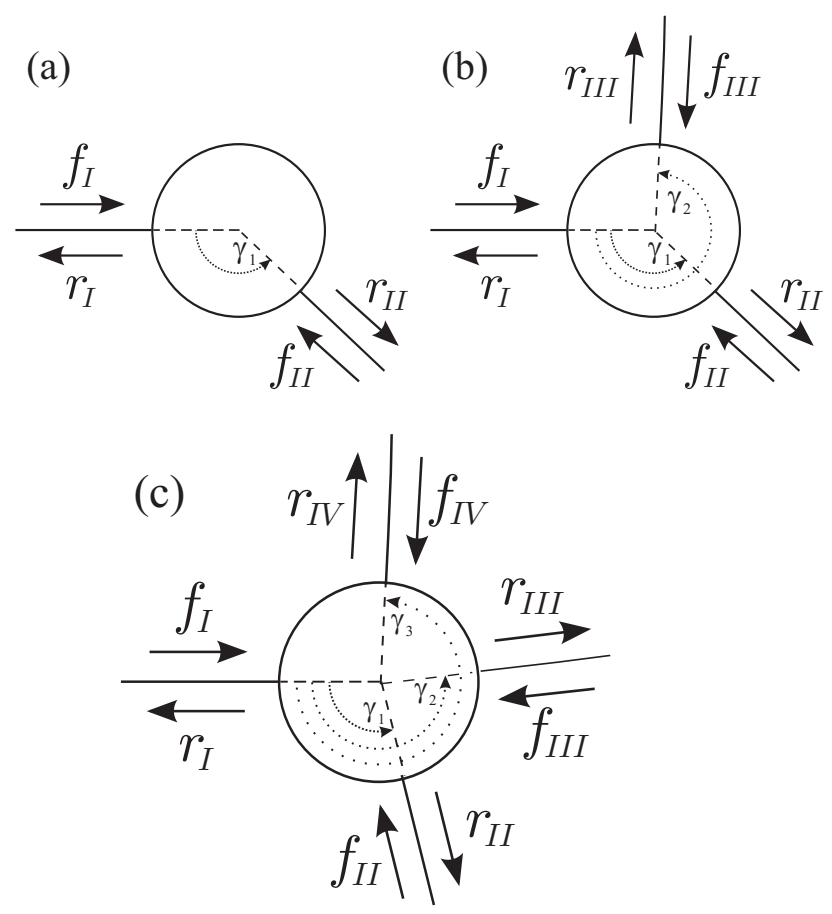

FIG. 1: The notations used for the spinor part of the wave functions in the case of two- (a) three- (b) and four-terminal rings (c)

where

$$
\begin{aligned}
\varrho^{(\mu)}= & 8 k a / y^{(\mu)}\left\{-i\left(q^{(\mu)}\right)^{2} \sin \left(2 q^{(\mu)} \pi\right)\right. \\
& -k a q^{(\mu)}\left[\sin \left(q^{(\mu)} \gamma_{1}\right) \sin \left(q^{(\mu)}\left(2 \pi-\gamma_{1}\right)\right)\right. \\
& \left.+\sin \left(q^{(\mu)} \gamma_{2}\right) \sin \left(q^{(\mu)}\left(2 \pi-\gamma_{2}\right)\right)\right] \\
& +i k^{2} a^{2} \sin \left(q^{(\mu)} \gamma_{1}\right) \sin \left(q^{(\mu)}\left(\gamma_{2}-\gamma_{1}\right)\right) \\
& \left.\times \sin \left(q^{(\mu)}\left(2 \pi-\gamma_{2}\right)\right)\right\}
\end{aligned}
$$

and

$$
\begin{aligned}
y^{(\mu)}= & 8\left(q^{(\mu)}\right)^{3}\left[\cos \left[\left((-1)^{\mu+1} w+2 \phi\right) \pi\right]+\cos \left(2 q^{(\mu)} \pi\right)\right] \\
& -12 i k a\left(q^{(\mu)}\right)^{2} \sin \left(2 q^{(\mu)} \pi\right)+4 k^{2} a^{2} q^{(\mu)} \cos \left(2 q^{(\mu)} \pi\right) \\
& -2 k^{2} a^{2} q^{(\mu)}\left[\cos \left(2 q^{(\mu)}\left(\pi-\gamma_{2}+\gamma_{1}\right)\right)-\cos \left(2 q^{(\mu)} \pi\right)\right. \\
& \left.+\cos \left(2 q^{(\mu)}\left(\pi-\gamma_{2}\right)\right)+\cos \left(2 q^{(\mu)}\left(\pi-\gamma_{1}\right)\right)\right] \\
& +i k^{3} a^{3}\left[\sin \left(2 q^{(\mu)}\left(\pi-\gamma_{2}+\gamma_{1}\right)\right)-\sin \left(2 q^{(\mu)} \pi\right)\right. \\
& \left.+\sin \left(2 q^{(\mu)}\left(\pi-\gamma_{1}\right)\right)-\sin \left(2 q^{(\mu)}\left(\pi-\gamma_{2}\right)\right)\right]
\end{aligned}
$$

with $\phi=\Phi / \Phi_{0}$. The matrices describing the connection between the outgoing spinors $r_{I I}, r_{I I I}$ and the input $f_{I}-$ the so called transmission matrices - are given by

$$
\begin{aligned}
& \left(T_{n}^{f_{I}}\right)_{\uparrow \uparrow}=e^{-i \gamma_{n} / 2}\left(\tau_{n}^{(1)} \cos ^{2}(\theta / 2)+\tau_{n}^{(2)} \sin ^{2}(\theta / 2)\right), \\
& \left(T_{n}^{f_{I}}\right)_{\uparrow \downarrow}=e^{-i \gamma_{n} / 2}\left(\tau_{n}^{(1)}-\tau_{n}^{(2)}\right) \sin (\theta / 2) \cos (\theta / 2), \\
& \left(T_{n}^{f_{I}}\right)_{\downarrow \uparrow}=e^{i \gamma_{n} / 2}\left(\tau_{n}^{(1)}-\tau_{n}^{(2)}\right) \sin (\theta / 2) \cos (\theta / 2), \\
& \left(T_{n}^{f_{I}}\right)_{\downarrow \downarrow}=e^{i \gamma_{n} / 2}\left(\tau_{n}^{(1)} \sin ^{2}(\theta / 2)+\tau_{n}^{(2)} \cos ^{2}(\theta / 2)\right),
\end{aligned}
$$

where $n=1,2$, indicating the two possible output channels and

$$
\begin{aligned}
\tau_{1}^{(\mu)}= & \frac{8 k a q^{(\mu)}}{y^{(\mu)}} e^{i \gamma_{1} / 2\left((-1)^{\mu+1} w+2 \phi\right)} \times \\
& \left\{-k a \sin \left(q^{(\mu)}\left(\gamma_{2}-\gamma_{1}\right)\right) \sin \left(q^{(\mu)}\left(2 \pi-\gamma_{2}\right)\right)\right. \\
& +i q^{(\mu)}\left[e^{-i \pi\left((-1)^{\mu+1} w+2 \phi\right)} \sin \left(q^{(\mu)} \gamma_{1}\right)\right. \\
& \left.\left.-\sin \left(q^{(\mu)}\left(2 \pi-\gamma_{1}\right)\right)\right]\right\}, \\
\tau_{2}^{(\mu)}= & \frac{8 k a q^{(\mu)}}{y^{(\mu)}} e^{i \gamma_{2} / 2\left((-1)^{\mu+1} w+2 \phi\right)} \times \\
& \left\{k a e^{-i \pi\left((-1)^{\mu+1} w+2 \phi\right)} \sin \left(q^{(\mu)} \gamma_{1}\right) \sin \left(q^{(\mu)}\left(\gamma_{2}-\gamma_{1}\right)\right)\right. \\
& +i q^{(\mu)}\left[e^{-i \pi\left((-1)^{\mu+1} w+2 \phi\right)} \sin \left(q^{(\mu)} \gamma_{2}\right)\right. \\
& \left.\left.-\sin \left(q^{(\mu)}\left(2 \pi-\gamma_{2}\right)\right)\right]\right\} .
\end{aligned}
$$

Note that the boundary conditions applied to obtain the $R^{f_{I}}$ and $T_{n}^{f_{I}}$ matrices above are similar to that of Ref. 35 . However the magnetic field induced shift of the spin Zeeman levels leads to a doubling of the parameters according to Eq. (4). This modifies significantly the physical transport properties of the device.

Let us point out that having obtained the matrix elements above is enough to handle the problem with both incoming and outgoing waves on all terminals of the ring as shown in Fig. 1(b). Namely, we can consider the three inputs $f_{i}(i=I, I I, I I I)$ separately and determine the corresponding reflection and transmission matrices. The outputs in the superposed problem will consist of contributions from all inputs: the reflected part of the spinor which enters on the same lead, and the transmitted parts of the other two inputs into the respective lead:

$$
\begin{aligned}
r_{I} & =R^{f_{I}} f_{I}+T_{2}^{f_{I I}} f_{I I}+T_{1}^{f_{I I I}} f_{I I I}, \\
r_{I I} & =T_{1}^{f_{I}} f_{I}+R^{f_{I I}} f_{I I}+T_{2}^{f_{I I I}} f_{I I I}, \\
r_{I I I} & =T_{2}^{f_{I}} f_{I}+T_{1}^{f_{I I}} f_{I I}+R^{f_{I I I}} f_{I I I} .
\end{aligned}
$$

Considering $f_{I I}\left(f_{I I I}\right)$ as the only input, the reflection and transmission matrices are the same as those for the input $f_{I}$, except for the appropriate changes of the angles, since in the reference frame of $f_{I I}\left(f_{I I I}\right)$, the angles of the output leads are measured from the lead through which $f_{I I}\left(f_{I I I}\right)$ enters the ring. In order to get the contributions to the output spinors for the input $f_{I I}\left(f_{I I I}\right)$ in 
the reference frame of $f_{I}$, the matrices need to be rotated (see Fig. 1(b) by the angle of $\gamma_{1}\left(\gamma_{2}\right)$ :

$$
\begin{gathered}
M^{f_{I I}}=U_{\gamma_{1}} M_{\substack{\gamma_{1} \leftrightarrow \gamma_{2}-\gamma_{1} \\
\gamma_{2} \leftrightarrow 2 \pi-\gamma_{1}}}^{f_{I}} U_{\gamma_{1}}^{-1} \\
M^{f_{I I I}}=U_{\gamma_{2}} M_{\substack{\gamma_{1} \leftrightarrow 2 \pi-\gamma_{2} \\
\gamma_{2} \leftrightarrow 2 \pi-\gamma_{2}+\gamma_{1}}}^{f_{I}} U_{\gamma_{2}}^{-1}
\end{gathered}
$$

where $M=R, T_{1}, T_{2}$ and

$$
U_{\gamma_{n}}=\left(\begin{array}{cc}
e^{-i \frac{\gamma_{n}}{2}} & 0 \\
0 & e^{i \frac{\gamma_{n}}{2}}
\end{array}\right), \quad n=1,2
$$

The above approach is also valid in the case of the twoand four-terminal rings. Using the reflection and transmission matrices as presented in the Appendix, the more general problem of having both incoming and outgoing waves on all terminals can easily be treated. All possible reflections and transmissions can thus be taken into account when forming two-dimensional arrays of such rings.

\section{RECTANGULAR ARRAYS OF QUANTUM RINGS}

\section{A. Magnetoconductance properties}

Based on the analytic results presented in the previous section and in the Appendix we may build $\mathrm{N} \times \mathrm{M}$ two-dimensional rectangular arrays of quantum rings, where both perpendicular electric and magnetic fields are present, so that the former one can be used to change the strength of the SOI 3 Here we focus on of $3 \times 3,4 \times 4$, and $5 \times 5$ arrays and assume that neighboring rings touch each other. In addition, we limit ourselves to arrays that are closed in the vertical, and open in the horizontal direction, as shown in Fig. 2, Two types of such arrays will be investigated: i) the electron can enter/exit the array through any of the rings in the horizontal direction, ii) the electron can enter the array through one ring only (no leads are attached to the other rings on the entrance side), but can exit through any of the rings on the opposite side (Fig. 2 without the dashed curves). In both cases the conductance is derived from the linear set of equations resulting from the fit of the wave functions $\Psi_{i}^{(k l)}$ ( $i=I, I I, I I I, I N$ and $k, l=1 . . N$, where $N$ is the number of rings along one direction in the array) and their derivatives $\partial_{x_{i}^{(k l)}} \Psi_{i}^{(k l)}$ in the points, where the rings touch each other, i.e. for example:

$$
\begin{aligned}
\left.\Psi_{I I I}^{(11)}\right|_{x_{I I I}^{(11)}=0} & =\left.\Psi_{I}^{(12)}\right|_{x_{I}^{(12)}=0}, \\
\left.\partial_{x_{I I I}^{(11)}} \Psi_{I I I}^{(11)}\right|_{x_{I I I}^{(11)}=0} & =-\left.\partial_{x_{I}^{(12)}} \Psi_{I}^{(12)}\right|_{x_{I}^{(12)}=0}
\end{aligned}
$$

Here we used the notations of Fig. 2 (Note that the negative sign in Eq. (12) is a consequence of the opposite direction of the local coordinates in the leads $I I I$ of ring $\{11\}$ and $I$ of ring $\{12\}$ ) Eqs. (12) lead to

$$
\begin{aligned}
& f_{I I I}^{(11)}+r_{I I I}^{(11)}=f_{I}^{(12)}+r_{I}^{(12)}, \\
& f_{I I I}^{(11)}-r_{I I I}^{(11)}=-f_{I}^{(12)}+r_{I}^{(12)}
\end{aligned}
$$

from which follows that

$$
\begin{aligned}
& f_{I I I}^{(11)}=r_{I}^{(12)} \\
& r_{I I I}^{(11)}=f_{I}^{(12)}
\end{aligned}
$$

i.e., the spinor entering (exiting) ring $\{11\}$ on terminal $I I I$ is equal to the spinor exiting (entering) ring $\{12\}$ on terminal $I$. The spinors $r_{I I I}^{(11)}$ and $r_{I}^{(12)}$ can be given with the help of the reflection and transmission matrices of a three-terminal ring according to Eqs. (9).

For a small number of rings the resulting set of equations can be solved analytically, however already for an array of $3 \times 3$ rings shown in Fig. 2, it consists of 60 equations, which is preferably solved by numerical means, although analytic solutions exist in principle. (For larger arrays the number of equations scales practically with the number of rings). After having determined the output spinor valued wave functions $r_{I I I}^{(1 N)}, r_{I I I}^{(2 N)}, \ldots r_{I I}^{(N N)}$, where $N$ is the number of rings in the horizontal direction, the Landauer-Büttiker ${ }^{29}$ formula

$$
G=G_{\uparrow}+G_{\downarrow}
$$

where

$$
\begin{aligned}
& G_{\uparrow}=\frac{e^{2}}{h}\left(\left|\left(r_{I I I}^{(1 N)}\right)_{\uparrow}\right|^{2}+\left|\left(r_{I I I}^{(2 N)}\right)_{\uparrow}\right|^{2}+\ldots+\left|\left(r_{I I}^{(N N)}\right)_{\uparrow}\right|^{2}\right), \\
& G_{\downarrow}=\frac{e^{2}}{h}\left(\left|\left(r_{I I I}^{(1 N)}\right)_{\downarrow}\right|^{2}+\left|\left(r_{I I I}^{(2 N)}\right)_{\downarrow}\right|^{2}+\ldots+\left|\left(r_{I I}^{(N N)}\right)_{\downarrow}\right|^{2}\right),
\end{aligned}
$$

is used to calculate the conductance of the arrays, averaged over the two $\sigma_{z}$ eigenspinor inputs. We note that our method of using single rings as building blocks can easily be used to determine the conductance of arrays of arbitrary - not necessarily rectangular - configuration as well.

Figure 3 shows a contour plot of the conductance (in $e^{2} / h$ units) of rectangular arrays of $3 \times 3,4 \times 4$ and $5 \times 5$ quantum rings, for zero magnetic flux as a function of the SOI strength $\omega_{\mathrm{SO}} / \Omega$ and $k a$. The values of $k a$ are varied around $k_{F} a=20.4$, corresponding to a Fermi energy $11.13 \mathrm{meV}$ in case of an effective mass $m^{*}=0.023 \mathrm{~m}$ of InAs and rings of radius $a=0.25 \mu \mathrm{m}$. In two-dimensional electron systems within an InAs quantum well, the value of $\alpha$ can be varied ${ }^{2,3}$ up to 40 peVm. The different arrays show similar behavior for larger values of the SOI strength: there are slightly downwards bending stripes (initially around even values of $k a$ ) where the devices are completely opaque for the electrons, and also conducting regions which are initially around odd values of $k a$ and have complex internal structure. Comparing our results to the case of a single ring with diametrically coupled 


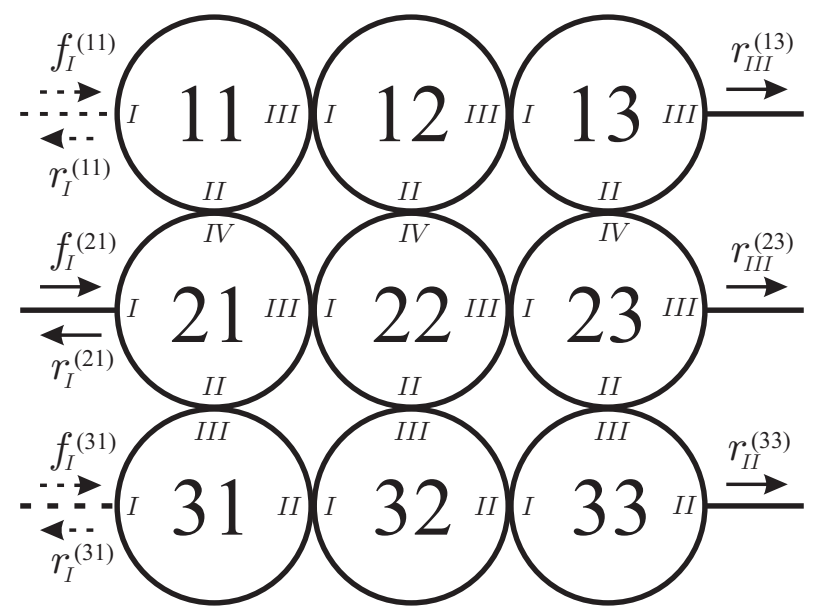

FIG. 2: The geometry of the device in the simplest case of a $3 \times 3$ array with three or one (without leads displayed with dashed lines) input terminals. The notations can easily be generalized to larger arrays.

leads 11 , it can be seen that the overall periodicity as a function of $k a$ is determined by single-ring interferences. The increasing number of the rings causes modulations superimposing on the single-ring behavior. This point is probably the most apparent if we recall ${ }^{11}$ that zero conductance areas are simply lines on the $k a-\omega_{\mathrm{SO}} / \Omega$ plane for a single two-terminal ring, while in our case there are stripes, the width of which is slightly increasing with the size of the array. This effect is related to the increasing number of consecutive partially destructive interferences that finally lead to essentially zero currents at the outputs. Additionally, if we considered an infinite network, the periodic boundary conditions would allow only discrete values of $k a$ for a given SOI strength with nonzero conductance. Thus the results presented in Fig. 3 demonstrate a transition between the conductance properties of a single ring and that of an infinite network.

Focusing on small values of $\omega_{\mathrm{SO}} / \Omega$, Fig. 3 shows a narrowing of the non-conducting regions until they eventually disappear when no SOI is present. Here the conductance still depends on $k a$, but its minimal values are not zeros and a periodic behavior can be seen: for a network of $N \times N$ rings, there are $N$ minima as the value of $k a$ is increased by 1 . This size-dependent modulation is related to the horizontal extent of the device: If we compare the conductance of the networks to that of rings of the same size and number without vertical connections, the same periodic behavior can be seen around zero SOI.

Figure 4 shows the normalized magnetoconductance of networks of $3 \times 3,4 \times 4$ and $5 \times 5$ quantum rings for $k a=19.6$ as a function of the SOI strength and the magnetic flux $\Phi$ (measured in units of $\Phi_{0}$ ). When $\omega_{\mathrm{SO}} / \Omega$ is zero, Aharonov-Bohm (AB) oscillations appear. For larger values of $\omega_{\mathrm{SO}} / \Omega$ both $\mathrm{AB}$ and Aharonov-Casher ${ }^{28}$ oscillations can be seen in the magnetoconductance. As Fig. 4 was plotted for a certain value of $k a$, the effect of the bending non-conducting stripes seen in Fig. 3 can
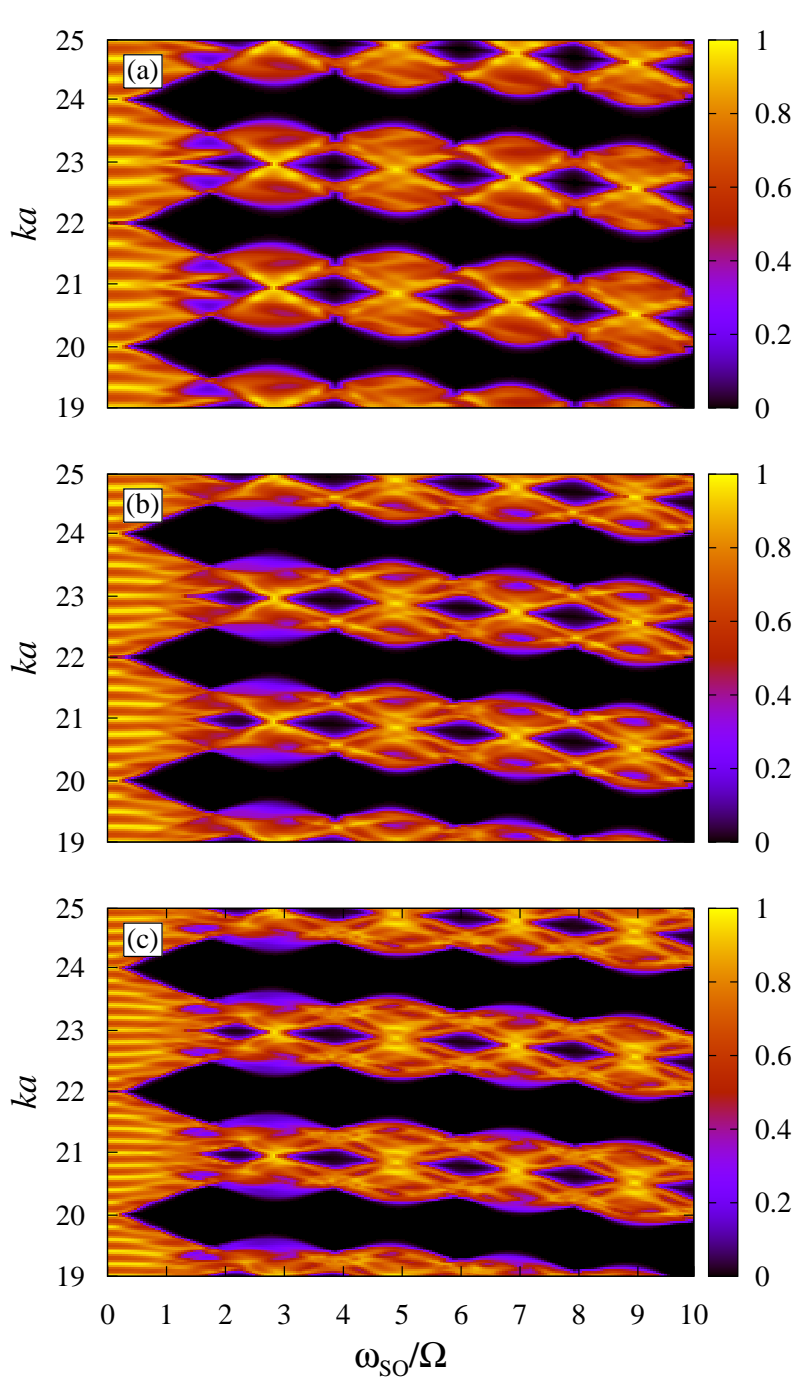

FIG. 3: (color online) The conductance $G / G_{0}\left(G_{0}=e^{2} / h\right)$ of a $3 \times 3$ (a) $4 \times 4(\mathrm{~b})$ and $5 \times 5$ (c) rectangular array with 3,4 , and 5 input terminals, respectively, for zero magnetic flux as a function of the SOI strength and $k a$.

also be seen as the decrease of the conductance when such a stripe is reached due to the change of the SOI strength, and its increase again, when the stripe is left. We note that for larger values of $k a$ this bending effect is less pronounced.

Figures 5 and 6 show the conductance of a $5 \times 5$ network with a single input lead in the middle (i.e. attached to ring $\{31\}$, using the notations of Fig. 2) as a function of $k a$, and $\omega_{\mathrm{SO}} / \Omega$ (Fig. 5), and the magnetic field and $\omega_{\mathrm{SO}} / \Omega$ (Fig. 6). The overall structure of these plots remains the same as in the case when the current can enter through all the rings on the left hand side, but the different boundary conditions modify the fine structure of the plots.

Our method allows the calculation of the spin directions for the different output terminals, and we found that spin-dependent interference in the array results in 

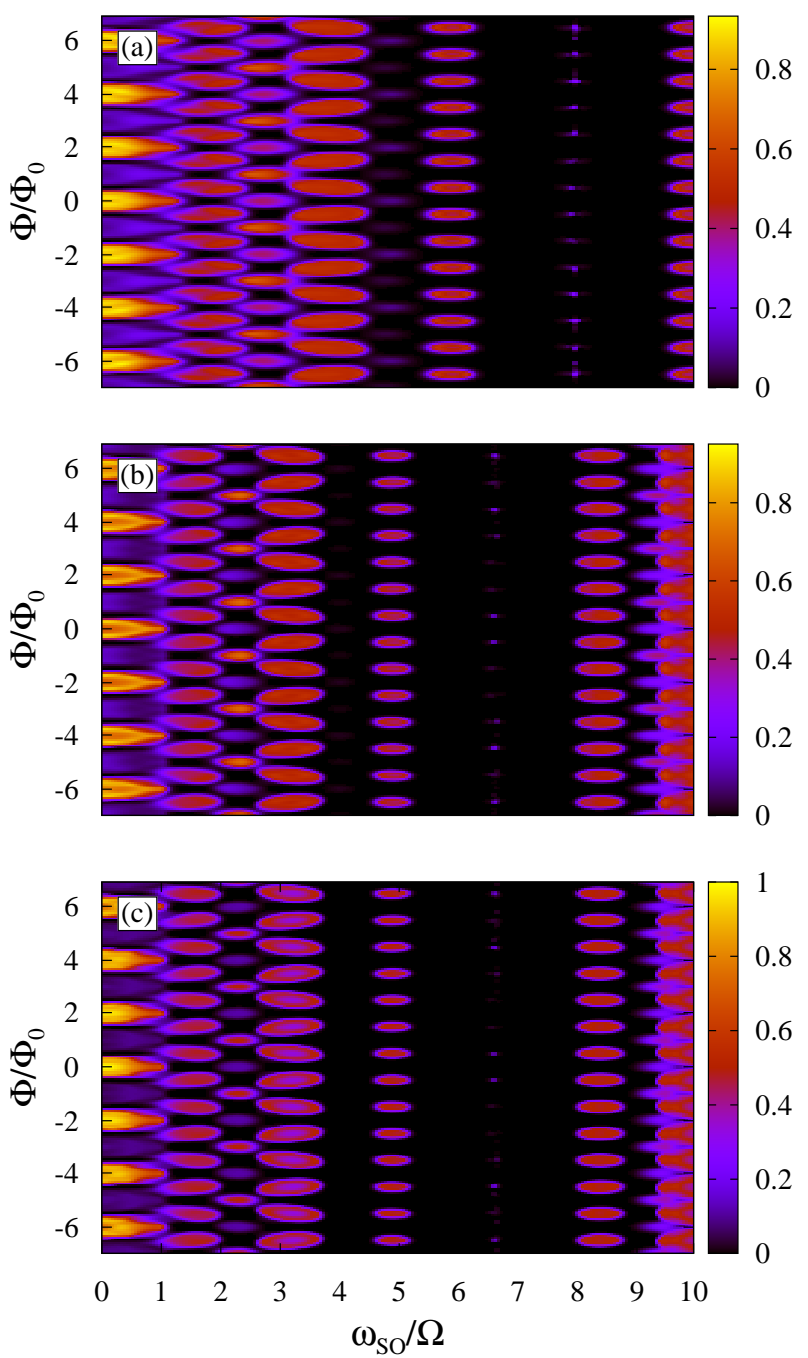

FIG. 4: (color online) The conductance $G / G_{0}\left(G_{0}=e^{2} / h\right)$ of a $3 \times 3$ (a) $4 \times 4$ (b) and $5 \times 5$ (c) rectangular array with 3 , 4, and 5 input terminals, respectively, for $k a=19.6$ as a function of the SOI strength and the magnetic flux $\Phi$ (in units of $\left.\Phi_{0}=h / e\right)$.

nontrivial spin transformations. Fig. 7 shows the spin resolved transmission probabilities for a $5 \times 5$ ring array with a single input lead. The incoming spin state is chosen to be $\left|\uparrow_{z}\right\rangle$, i.e., the spin-up eigenstate of $\sigma_{z}$, and the contour plots show the probabilities of the $\left|\uparrow_{x}\right\rangle,\left|\uparrow_{y}\right\rangle$ and $\left|\uparrow_{z}\right\rangle$ outputs at ring $\{55\}$ on the right hand side. The fact that the $\left|\uparrow_{z}\right\rangle$ input spinor changes its direction (as it is seen in Fig. 7, it can be transformed into $\left|\uparrow_{x}\right\rangle$ or $\left.\left|\uparrow_{y}\right\rangle\right)$ is due to the SOI induced spin rotations. The actual values of the spin resolved transmission probabilities are determined by the spin dependent interference phenomena. Fig. 8 shows the $z$ component of the normalized output spinors and visualizes that spin resolved results depend on the input side geometry as well. As we can see, the spin components change in the whole available range between -1 and 1 , and their behavior is rather dif-

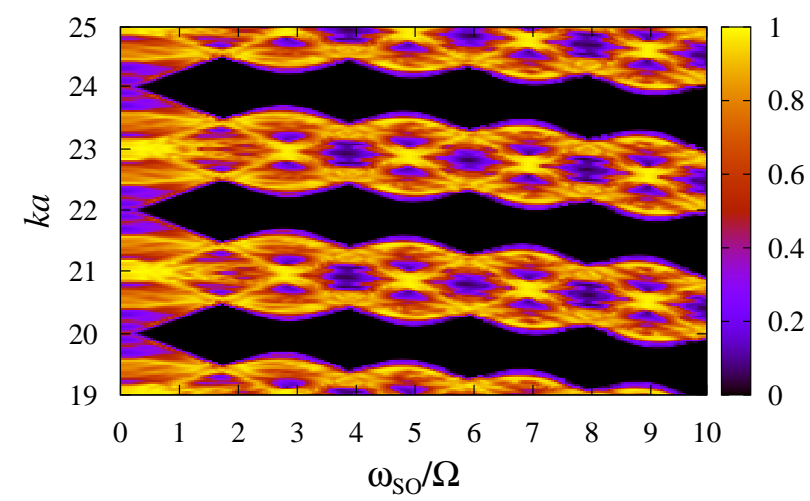

FIG. 5: (color online) The conductance $G / G_{0}\left(G_{0}=e^{2} / h\right)$ of a $5 \times 5$ rectangular array with a single input lead attached to ring $\{31\}$, for zero magnetic flux as a function of the SOI strength and $k a$.

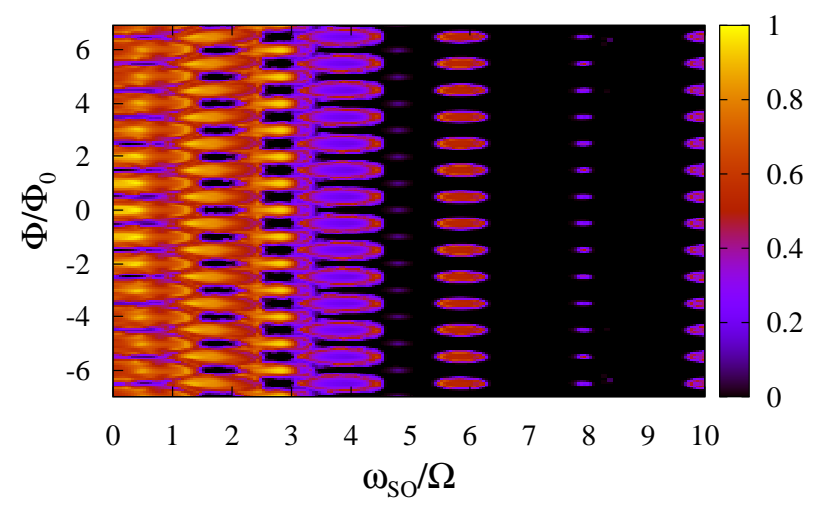

FIG. 6: (color online) The conductance $G / G_{0}\left(G_{0}=e^{2} / h\right)$ of a $5 \times 5$ rectangular array with a single input lead attached to ring $\{31\}$, for $k a=19.57$ as a function of the SOI strength and the magnetic flux $\Phi$ (in units of $\Phi_{0}=h / e$ ).

ferent for the cases when the electron can enter the array through any of the five terminals, or only through the one attached to ring $\{31\}$. This phenomenon together with other spin dependent interference effects $38,39,40,41,42,43,44$ can lead to spin sensitive quantum networks.

\section{B. Effect of point-like scatterers}

Now we will investigate to what extent the conductance properties are modified by the presence of random scatterers. Although high mobility samples have already become available (such that at cryogenic temperatures transport is found to be ballistic over tens of microns), considering also the effects caused by scattering events provides a more realistic description for most cases. To this end we introduce point-like scattering centers between the rings. In particular, at each point $j$ where two rings touch each other, we consider an ad- 

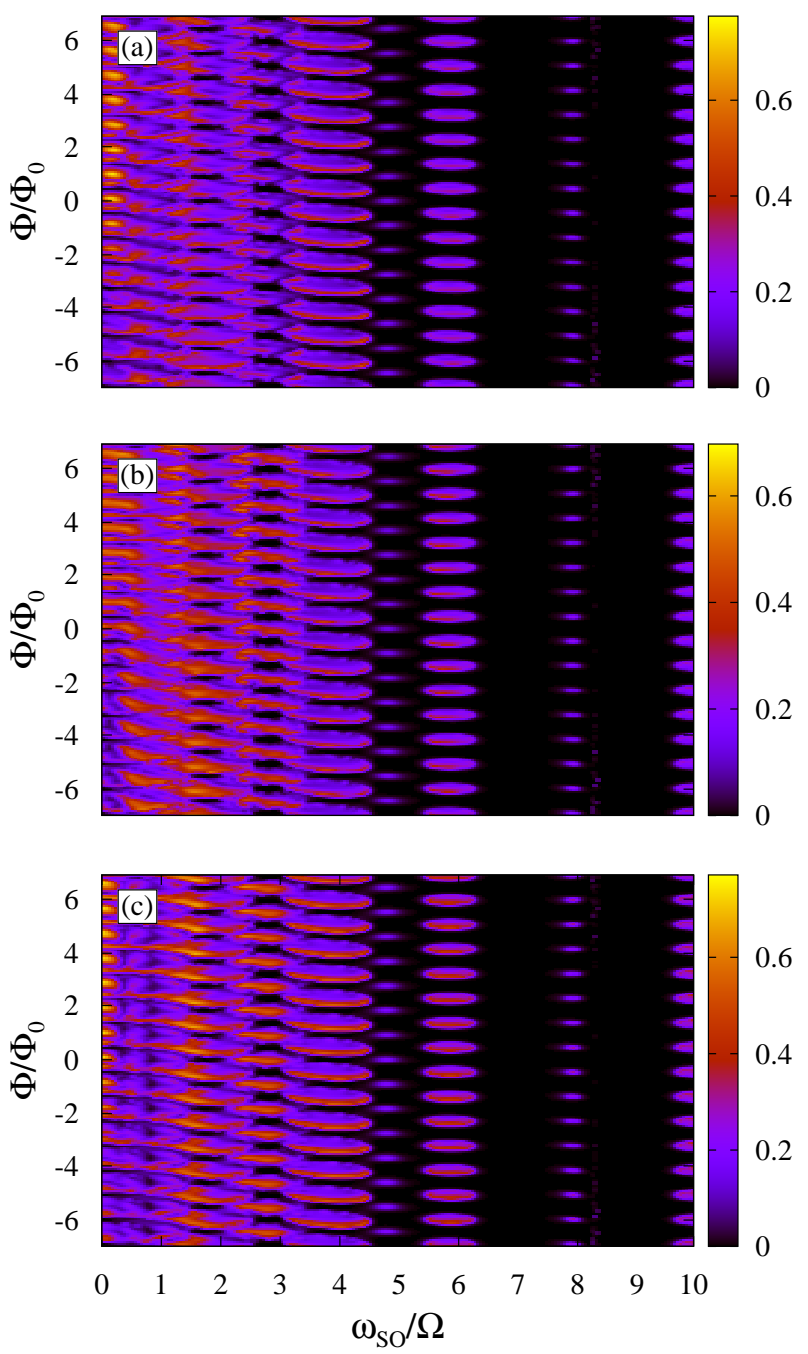

FIG. 7: (color online) The probabilities of the $\left|\uparrow_{x}\right\rangle(\mathrm{a})\left|\uparrow_{y}\right\rangle$ (b) and $\left|\uparrow_{z}\right\rangle(\mathrm{c})$ outputs at ring $\{55\}$ of a $5 \times 5$ rectangular array with one input lead (attached to ring $\{31\}$ ), for $k a=$ 19.6 as a function of the SOI strength and the magnetic flux $\Phi$ (in units of $\Phi_{0}=h / e$ ). The incoming spin state is chosen to be $|\uparrow z\rangle$.

ditional Dirac delta potential of the form $\eta_{j} \delta(j)$. Here $\eta_{j}$ represent independent normally distributed random variables with zero mean, and root-mean-square deviation $D$. By tuning $D$ we can model weak disturbances (small $D$ ) as well as the case when frequent scattering events completely change the character of the transport process (corresponding to large values of $D$ ).

As shown in Fig. 9, the most general consequence of these random scattering events is the overall decrease of the conductance. However, for strong enough disturbance, more interesting effects can be seen, namely the splitting of the $\mathrm{AB}$ peaks which is more pronounced with increasing $D$. Note that the scattering has the most dramatic effect for the $\mathrm{AB}$ resonances, i.e. $\Phi=n \Phi_{0}$ and the least for the anti-resonance condition, i.e. $\Phi=$

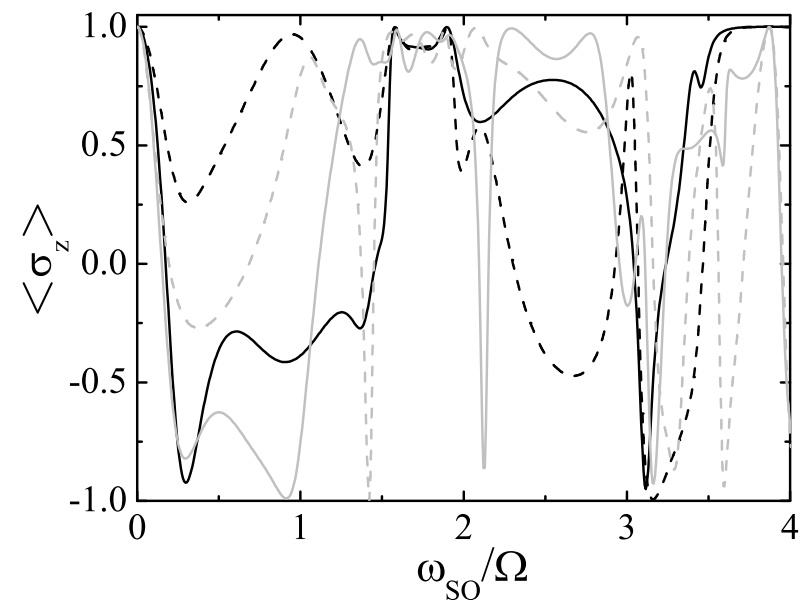

FIG. 8: The spin transformation properties of a $5 \times 5$ array with input leads attached to all rings and only to ring $\{31\}$ (black and grey curves, respectively). The $z$ component of the normalized spin states transmitted via the output terminals attached to ring $\{25\}$ (solid line) and ring $\{45\}$ (dashed line). The incoming spin state is chosen to be $\left|\uparrow_{z}\right\rangle$.

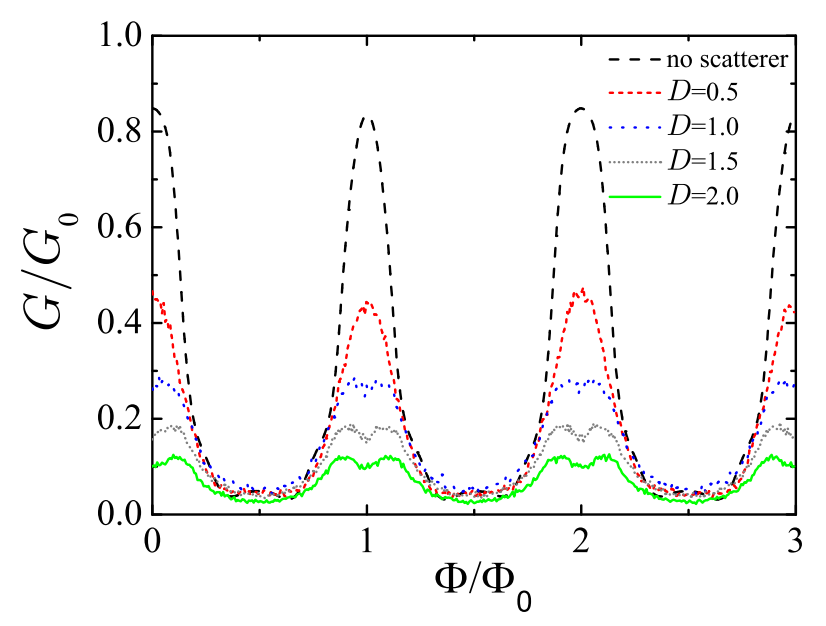

FIG. 9: (color online) The conductance $G$ (in units of $G_{0}=$ $\left.e^{2} / h\right)$ of a $5 \times 5$ rectangular array with point-like random scatterers between the rings for different root-mean-square deviations $D$ as a function of the magnetic flux $\Phi$ (in units of $\left.\Phi_{0}=h / e\right)$ for $k a=20.2$ and $\omega_{\mathrm{SO}} / \Omega=13.0$.

$(n+1 / 2) \Phi_{0}$. Fig. 10 shows that the conductance has an oscillatory dependence on the strength of the SOI. Notice that the introduction of scattering reduces the periodicity with a factor of two. We want to stress that the model we considered (random elastic scattering processes in single-electron approximation) is similar to the case when the Altshuler-Aronov-Spivak (AAS) effect $\frac{45}{5}$ is expected to survive in a single ring. Our results for a more complex geometry indicate similar physical consequences of the scattering events: introduction of new peaks in the $\mathrm{AB}$ oscillations and appearance of $\omega_{\mathrm{SO}} / \Omega$ 


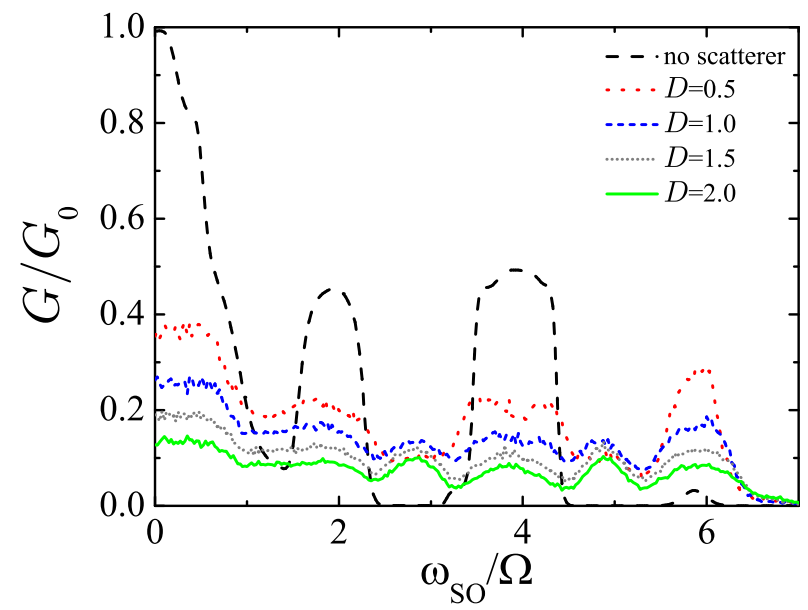

FIG. 10: (color online) The conductance $G$ (in units of $G_{0}=e^{2} / h$ ) of a $5 \times 5$ rectangular array with point-like random scatterers between the rings for different root-mean-square deviations $D$ as a function of the SOI strength for $k a=19.6$ and $\Phi=0.3 \Phi_{0}$.

conductance oscillations for a given value of $\Phi$. This latter effect has recently been predicted for a single ring 46 and was detected in the case of ring arrays $\underline{26}^{26}$

\section{SUMMARY}

In this paper we calculated the spin dependent transport properties of two-dimensional ring arrays. We applied general boundary conditions for the case of single quantum rings, which allowed the construction of arrays of such rings as building blocks. The magnetoconductance of two-dimensional arrays of $3 \times 3,4 \times 4$, and $5 \times 5$ quantum rings exhibited Aharonov-Bohm and AharonovCasher oscillations. We also determined the spin resolved transmission probabilities of the arrays and found significant spin rotations depending on the SOI strength. We introduced point-like random scattering centers between the rings, which, for strong enough disturbance resulted in the splitting of the $\mathrm{AB}$ peaks and the emergence of an oscillatory behavior of the conductance, characteristic to the SOI strength.

\section{Acknowledgments}

This work was supported by the Flemish-Hungarian Bilateral Programme, the Flemish Science Foundation (FWO-Vl), the Belgian Science Policy and the Hungarian Scientific Research Fund (OTKA) under Contracts Nos. T48888, M36803, M045596. P.F. was supported by a J. Bolyai grant of the Hungarian Academy of Sciences. We thank J. Sólyom for enlightening discussions.

\section{Appendix}

Here we present the detailed analytic expressions of the scattering problem for a general two- and four-terminal ring, in which SOI and a perpendicular magnetic field is present, the latter of which is considered as a perturbation. As we have shown in Sec. II it is sufficient to consider only one input terminal and determine the connection between the input and output states, i.e. the reflection and transmission matrices, since the more general boundary condition of having inputs on all terminals is just a superposition of such cases with an appropriate rotation of the matrices (see Eqs. (10)-(11)). Considering $f_{I}$ as the only input (i.e. $f_{i \neq I}=0$, in Figs. 1(a) and $1(\mathrm{c})$, requiring the continuity of the wave functions and applying Griffith boundary conditions ${ }^{32.37}$ at the junctions in both cases, we can obtain the reflection matrices $\hat{R}^{f_{I}}$ and $\tilde{R}^{f_{I}}$ of the two-terminal ring, and of the fourterminal ring, respectively. Both can be written in a form analogous to that of $R^{f_{I}}$ of the three terminal case given by Eq. (7) with

$$
\begin{aligned}
\hat{\varrho}^{(\mu)}= & \frac{4 k^{2} a^{2}}{\hat{y}^{(\mu)}}\left\{\sin \left(q^{(\mu)} \gamma_{1}\right) \sin \left(q^{(\mu)}\left(2 \pi-\gamma_{1}\right)\right)\right. \\
& \left.+i q^{(\mu)} \sin \left(2 q^{(\mu)} \pi\right)\right\}
\end{aligned}
$$

and

$$
\begin{aligned}
\tilde{\varrho}^{(\mu)}= & \frac{2 k a}{\tilde{y}^{(\mu)}}\left\{k ^ { 3 } a ^ { 3 } \left[\cos \left(2 q^{(\mu)} \pi\right)+\cos \left(2 q^{(\mu)}\left(\pi-\gamma_{3}+\gamma_{2}-\gamma_{1}\right)\right)\right.\right. \\
& -\cos \left(2 q^{(\mu)}\left(\pi-\gamma_{3}+\gamma_{2}\right)\right)+\cos \left(2 q^{(\mu)}\left(\pi-\gamma_{3}+\gamma_{1}\right)\right) \\
& -\cos \left(2 q^{(\mu)}\left(\pi-\gamma_{2}+\gamma_{1}\right)\right)-\cos \left(2 q^{(\mu)}\left(\pi-\gamma_{3}\right)\right) \\
& \left.+\cos \left(2 q^{(\mu)}\left(\pi-\gamma_{2}\right)\right)-\cos \left(2 q^{(\mu)}\left(\pi-\gamma_{1}\right)\right)\right] \\
& +2 i k^{2} a^{2} q^{(\mu)}\left[\sin \left(2 q^{(\mu)}\left(\pi-\gamma_{3}+\gamma_{2}\right)\right)-3 \sin \left(2 q^{(\mu)} \pi\right)\right. \\
& \left.+\sin \left(2 q^{(\mu)}\left(\pi-\gamma_{3}+\gamma_{1}\right)\right)+\sin \left(2 q^{(\mu)}\left(\pi-\gamma_{2}+\gamma_{1}\right)\right)\right] \\
& +4 i k^{2} a^{2} q^{(\mu)}\left[\sin \left(2 q^{(\mu)}\left(\pi-\gamma_{1}\right)\right)-\sin \left(2 q^{(\mu)}\left(\pi-\gamma_{3}\right)\right)\right] \\
& -4 k a\left(q^{(\mu)}\right)^{2}\left[\cos \left(2 q^{(\mu)}\left(\pi-\gamma_{3}\right)\right)+\cos \left(2 q^{(\mu)}\left(\pi-\gamma_{2}\right)\right)\right. \\
& \left.+\cos \left(2 q^{(\mu)}\left(\pi-\gamma_{1}\right)\right)-3 \cos \left(2 q^{(\mu)} \pi\right)\right] \\
& \left.-8 i\left(q^{(\mu)}\right)^{3} \sin \left(2 q^{(\mu)} \pi\right)\right\},
\end{aligned}
$$

respectively. Here

$$
\begin{aligned}
\hat{y}^{(\mu)}= & k^{2} a^{2}\left[\cos \left(2 q^{(\mu)}\left(\pi-\gamma_{1}\right)\right)-\cos \left(2 q^{(\mu)} \pi\right)\right] \\
& +4 i k a q^{(\mu)} \sin \left(2 q^{(\mu)} \pi\right) \\
& -4\left(q^{(\mu)}\right)^{2}\left[\cos \left[\left((-1)^{\mu+1} w+2 \phi\right) \pi\right]+\cos \left(2 q^{(\mu)} \pi\right)\right],
\end{aligned}
$$




$$
\begin{aligned}
\tilde{y}^{(\mu)}= & 16\left(q^{(\mu)}\right)^{4}\left[\cos \left[\left((-1)^{\mu+1} w+2 \phi\right) \pi\right]+\cos \left(2 q^{(\mu)} \pi\right)\right] \\
& -32 i k a\left(q^{(\mu)}\right)^{3} \sin \left(2 q^{(\mu)} \pi\right)+24 k^{2} a^{2}\left(q^{(\mu)}\right)^{2} \cos \left(2 q^{(\mu)} \pi\right) \\
& -4 k^{2} a^{2}\left(q^{(\mu)}\right)^{2}\left[\cos \left(2 q^{(\mu)}\left(\pi-\gamma_{3}\right)\right)+\cos \left(2 q^{(\mu)}\left(\pi-\gamma_{2}\right)\right)\right. \\
& +\cos \left(2 q^{(\mu)}\left(\pi-\gamma_{1}\right)\right)+\cos \left(2 q^{(\mu)}\left(\pi-\gamma_{3}+\gamma_{1}\right)\right) \\
& \left.+\cos \left(2 q^{(\mu)}\left(\pi-\gamma_{3}+\gamma_{2}\right)\right)+\cos \left(2 q^{(\mu)}\left(\pi-\gamma_{2}+\gamma_{1}\right)\right)\right] \\
& -8 i k^{3} a^{3} q^{(\mu)} \sin \left(2 q^{(\mu)} \pi\right) \\
& +4 i k^{3} a^{3} q^{(\mu)}\left[\sin \left(2 q^{(\mu)}\left(\pi-\gamma_{3}+\gamma_{2}\right)\right)-\sin \left(2 q^{(\mu)}\left(\pi-\gamma_{3}\right)\right)\right. \\
& \left.+\sin \left(2 q^{(\mu)}\left(\pi-\gamma_{2}+\gamma_{1}\right)\right)+\sin \left(2 q^{(\mu)}\left(\pi-\gamma_{1}\right)\right)\right] \\
& +k^{4} a^{4}\left[\cos \left(2 q^{(\mu)}\left(\pi-\gamma_{3}+\gamma_{2}-\gamma_{1}\right)\right)+\cos \left(2 q^{(\mu)} \pi\right)\right. \\
& +\cos \left(2 q^{(\mu)}\left(\pi-\gamma_{3}+\gamma_{1}\right)\right)-\cos \left(2 q^{(\mu)}\left(\pi-\gamma_{3}+\gamma_{2}\right)\right) \\
& -\cos \left(2 q^{(\mu)}\left(\pi-\gamma_{2}+\gamma_{1}\right)\right)-\cos \left(2 q^{(\mu)}\left(\pi-\gamma_{3}\right)\right) \\
& \left.+\cos \left(2 q^{(\mu)}\left(\pi-\gamma_{2}\right)\right)-\cos \left(2 q^{(\mu)}\left(\pi-\gamma_{1}\right)\right)\right]
\end{aligned}
$$

where the angles $\gamma_{i}$ are defined in Fig. 1(a) and (c). The transmission matrices $\hat{T}^{f_{I}}$ of the two-terminal ring and $\tilde{T}_{n}^{f_{I}}(n=1,2,3)$ of the four-terminal ring, can be given in an analogous form to that of the transmission matrices $T_{n}^{f_{I}}$ of the three-terminal one given by Eq. (8) with

$$
\begin{aligned}
\hat{\tau}^{(\mu)}= & \frac{4 i k a q^{(\mu)}}{\hat{y}^{(\mu)}} e^{i \gamma_{1}\left((-1)^{\mu+1} w / 2+\phi\right)}\left[\sin \left(q^{(\mu)}\left(2 \pi-\gamma_{1}\right)\right)\right. \\
& \left.-e^{-i \pi\left((-1)^{\mu+1} w+2 \phi\right)} \sin \left(q^{(\mu)} \gamma_{1}\right)\right]
\end{aligned}
$$

and

$$
\begin{aligned}
\tilde{\tau}_{1}^{(\mu)}= & \frac{4 k a q^{(\mu)}}{\tilde{y}^{(\mu)}} e^{i \gamma_{1} / 2\left((-1)^{\mu+1} w+2 \phi\right)} \times \\
& \left\{i k ^ { 2 } a ^ { 2 } \left[\sin \left(q^{(\mu)}\left(2 \pi-2 \gamma_{3}+2 \gamma_{2}-\gamma_{1}\right)\right)\right.\right. \\
& \left.-\sin \left(q^{(\mu)}\left(2 \pi-\gamma_{1}\right)\right)+\sin \left(q^{(\mu)}\left(2 \pi-2 \gamma_{2}+\gamma_{1}\right)\right)\right] \\
& \left.-\sin \left(q^{(\mu)}\left(2 \pi-2 \gamma_{3}+\gamma_{1}\right)\right)\right] \\
& -2 k a q^{(\mu)}\left[\cos \left(q^{(\mu)}\left(2 \pi-2 \gamma_{2}+\gamma_{1}\right)\right)\right. \\
& \left.-2 \cos \left(q^{(\mu)}\left(2 \pi-\gamma_{1}\right)\right)+\cos \left(q^{(\mu)}\left(2 \pi-2 \gamma_{3}+\gamma_{1}\right)\right)\right] \\
& +4 i\left(q^{(\mu)}\right)^{2}\left[e^{-i \pi\left((-1)^{\mu+1} w+2 \phi\right)} \sin \left(q^{(\mu)} \gamma_{1}\right)\right. \\
& \left.\left.-\sin \left(q^{(\mu)}\left(2 \pi-\gamma_{1}\right)\right)\right]\right\}
\end{aligned}
$$

$$
\begin{aligned}
\tilde{\tau}_{2}^{(\mu)}= & \frac{4 k a q^{(\mu)}}{\tilde{y}^{(\mu)}} e^{i \gamma_{2} / 2\left((-1)^{\mu+1} w+2 \phi\right)} \times \\
& \left\{-2 k a q^{(\mu)}\left[e^{-i \pi\left((-1)^{\mu+1} w+2 \phi\right)} \cos \left(q^{(\mu)} \gamma_{2}\right)\right.\right. \\
& -e^{-i \pi\left((-1)^{\mu+1} w+2 \phi\right)} \cos \left(q^{(\mu)}\left(2 \gamma_{1}-\gamma_{2}\right)\right) \\
& \left.+\cos \left(q^{(\mu)}\left(2 \pi-2 \gamma_{3}+\gamma_{2}\right)\right)-\cos \left(q^{(\mu)}\left(2 \pi-\gamma_{2}\right)\right)\right] \\
& +4 i\left(q^{(\mu)}\right)^{2}\left[e^{-i \pi\left((-1)^{\mu+1} w+2 \phi\right)} \sin \left(q^{(\mu)} \gamma_{2}\right)\right. \\
& \left.\left.-\sin \left(q^{(\mu)}\left(2 \pi-\gamma_{2}\right)\right)\right]\right\},
\end{aligned}
$$$$
\tilde{\tau}_{3}^{(\mu)}=\frac{4 k a q^{(\mu)}}{\tilde{y}^{(\mu)}} e^{i \gamma_{3} / 2\left((-1)^{\mu+1} w+2 \phi\right)} \times
$$$$
\left\{i k ^ { 2 } a ^ { 2 } e ^ { - i \pi ( ( - 1 ) ^ { \mu + 1 } w + 2 \phi ) } \left[\sin \left(q^{(\mu)} \gamma_{3}\right)\right.\right.
$$$$
+\sin \left(q^{(\mu)}\left(2 \gamma_{1}-\gamma_{3}\right)\right)-\sin \left(q^{(\mu)}\left(2 \gamma_{2}-\gamma_{3}\right)\right)
$$$$
\left.+\sin \left(q^{(\mu)}\left(2 \gamma_{2}-2 \gamma_{1}-\gamma_{3}\right)\right)\right]
$$$$
-2 k a q^{(\mu)} e^{-i \pi\left((-1)^{\mu+1} w+2 \phi\right)}\left[2 \cos \left(q^{(\mu)} \gamma_{3}\right)\right.
$$$$
\left.-\cos \left(q^{(\mu)}\left(2 \gamma_{1}-\gamma_{3}\right)\right)-\cos \left(q^{(\mu)}\left(2 \gamma_{2}-\gamma_{3}\right)\right)\right]
$$$$
+4 i\left(q^{(\mu)}\right)^{2}\left[e^{-i \pi\left((-1)^{\mu+1} w+2 \phi\right)} \sin \left(q^{(\mu)} \gamma_{3}\right)\right.
$$$$
\left.\left.-\sin \left(q^{(\mu)}\left(2 \pi-\gamma_{3}\right)\right)\right]\right\},
$$

respectively.

3 J. Nitta, T. Akazaki, H. Takayanagi, and T. Enoki, Phys. Rev. Lett. 78, 1335 (1997).

${ }^{4}$ Y. Sato, S. G. T. Kita, and S. Yamada, J. Appl. Phys. 89, 8017 (2001).

5 J. Splettstoesser, M. Governale, and U. Zülicke, Phys. Rev. B 68, 165341 (2003).
* Electronic address: benedict@physx.u-szeged.hu

1 M. König, A. Tschetschetkin, E. M. Hankiewicz, J. Sinova V. Hock, V. Daumer, M. Schäfer, C. R. Becker, H. Buhmann, and L. W. Molenkamp, Phys. Rev. Lett. 96, 076804 (2006).

2 D. Grundler, Phys. Rev. Lett. 84, 6074 (2000). 
6 J. S. Sheng and K. Chang, Phys. Rev. B 74, 235315 (2006).

7 S. R. E. Yang, Phys. Rev. B 74, 075315 (2006).

${ }^{8}$ L. W. Yu, K. J. Chen, J. Song, J. Xu, W. Li, X. F. Li, J. M. Wang, and X. F. Huang, Phys. Rev. Lett. 98, 166102 (2007).

9 M. Büttiker, Y. Imry, and M. Y. Azbel, Phys. Rev. A 30, 1982 (1984)

10 J. Nitta, F. E. Meijer, and H. Takayanagi, Appl. Phys. Lett. 75, 695 (1999).

11 B. Molnár, F. M. Peeters, and P. Vasilopoulos, Phys. Rev. B 69, 155335 (2004).

12 D. Frustaglia and K. Richter, Phys. Rev. B 69, 235310 (2004).

13 P. Földi, B. Molnár, M. G. Benedict, and F. M. Peeters, Phys. Rev. B 71, 033309 (2005).

14 X. F. Wang and P. Vasilopoulos, Phys. Rev. B 72, 165336 (2005).

15 S. Souma and B. K. Nikolić, Phys. Rev. Lett. 94, 106602 (2005).

16 Y. K. Kato, R. C. Myers, A. C. Gossard, and D. D. Awschalom, Appl. Phys. Lett. 86, 162107 (2005).

17 S. Bellucci and P. Onorato, J. Phys.: Condens. Matter 19, 395020 (2007).

18 B. Szafran and F. M. Peeters, Phys. Rev. B 72, 165301 (2005).

19 P. Vasilopoulos, O. Kálmán, F. M. Peeters, and M. G. Benedict, Phys. Rev. B 75, 035304 (2007).

20 J. B. Yau, E. P. DePoortere, and M. Shayegan, Phys. Rev. Lett. 88, 146801 (2002).

21 D. Frustaglia, M. Hentschel, and K. Richter, Phys. Rev. Lett. 87, 256602 (2001).

22 G. Cohen, O. Hod, and E. Rabani, Phys. Rev. B 76, 235120 (2007).

23 B. Molnár, P. Vasilopoulos, and F. M. Peeters, Phys. Rev. B 72, 075330 (2005).

24 E. I. Rashba, Sov. Phys. Solid State 2, 1109 (1960).

25 T. Koga, J. Nitta, T. Akazaki, and H. Takayanagi, Phys. Rev. Lett. 89, 046801 (2002).

26 T. Bergsten, T. Kobayashi, Y. Sekine, and J. Nitta, Phys.
Rev. Lett. 97, 196803 (2006).

27 Z. Zhu, Y. Wang, K. Xia, X. C. Xie, and Z. Ma, Phys. Rev. B 76, 125311 (2007).

28 Y. Aharonov and A. Casher, Phys. Rev. Lett. 53, 319 (1984).

29 S. Datta, Electronic Transport in Mesoscopic Systems (Cambridge Univ. Press, Cambridge, 1995).

30 P. Földi, B. Molnár, M. G. Benedict, and F. M. Peeters, Phys. Rev. B 71, 033309 (2005).

31 A. G. Aronov and Y. B. Lyanda-Geller, Phys. Rev. Lett. 70, 343 (1993).

32 J. B. Xia, Phys. Rev. B 45, 3593 (1992).

33 P. Földi, O. Kálmán, M. G. Benedict, and F. M. Peeters, Phys. Rev. B 73, 155325 (2006).

34 O. Kálmán, P. Földi, and M. G. Benedict, Open Sys. and Inf. Dyn. 13, 455 (2006).

35 O. Kálmán, P. Földi, M. G. Benedict, and F. M. Peeters, Physica E 40, 567 (2008).

36 F. E. Meijer, A. F. Morpurgo, and T. M. Klapwijk, Phys. Rev. B 66, 033107 (2002).

37 S. Griffith, Trans. Faraday Soc. 49, 345 (1953).

38 A. Pályi and J. Cserti, Phys. Rev. B 76, 035331 (2007).

39 J. Cserti, A. Csordás, and U. Zülicke, Phys. Rev. B 70, 233307 (2004).

40 T. Koga, J. Nitta, and M. van Veenhuizen, Phys. Rev. B 70, 161302(R) (2004).

41 R. Ionicioiu and I. D'Amico, Phys. Rev. B 67, 041307(R) (2003).

42 U. Zülicke and A. I. Signal, Solid State Commun. 144, 529 (2007).

43 A. W. Cummings, R. Akis, and D. K. Ferry, Appl. Phys. Lett. 89, 172115 (2006).

44 S. Bellucci and P. Onorato, Phys. Rev. B 77, 165305 (2008).

45 B. L. Al'tshuler, A. G. Aronov, and B. F. Spivak, JETP Lett. 33, 94 (1981).

46 M. J. van Veenhuizen, T. Koga, and J. Nitta, Phys. Rev. B 73, 235315 (2006). 